\title{
Knowledge of HPV and cervical cancer among HIV-positive women in Lagos, Nigeria
}

\author{
Rose Anorlu*, Maymuna Adegbesan, Temitope Adaramewa \\ From $12^{\text {th }}$ International Conference on Malignancies in AIDS and Other Acquired Immunodeficiencies \\ (ICMAOI) \\ Bethesda, MD, USA. 26-27 April, 2010
}

\section{Background}

Cervical cancer is the second most common cancer among women in the world. In parts of Africa it is the most common cancer among women. The age-specific incident rate is in some parts of Africa. Persistent infection with high-risk human papillomavirus (HPV) is a necessary cause of cervical cancer and HIV is a known co-factor that enhances the oncogenic potential of HPV. Sub-Saharan Africa has the highest number of people living with HIV. In Nigeria there are about 4.5 million people living with HIV/AIDS; more than half are women. Women infected with HIV are at greater risk of persistent HPV infection and consequently at greater risk of the associated premalignant disease and cervical cancer. The objective of this study was to determine the knowledge of HIV-positive women on HPV and cervical cancer.

\section{Materials and methods}

Two-hundred women who attended an HIV clinic in Lagos in January 2010 and who consented to participate in the study were randomly selected. They were given self-administered questionnaires. The questionnaire sought to determine their sociodemographic characteristics and their knowledge of HPV, cervical cancer, and the HPV vaccine.

\section{Results}

183 completed the questionnaire. The mean age was $33.0 \pm 6.1$ years (range 23-52 years). About one third (32.1\%) had had post-secondary school education and almost a third were single. $117(71.1 \%)$ had never been on any form of contraception.127 (69.3\%) had had the HIV test because they were very sick; only $16.7 \% \mathrm{had}$ voluntary testing. The awareness of cervical cancer was poor. $74.4 \%$ had never had any form of cervical cancer screening. About $90 \%$ had never heard of HPV and 92.3\% did not know that HPV causes cervical cancer, $82.1 \%$ did not know that HPV is contracted via sexual intercourse, and only $17.1 \%$ knew cervical cancer can be prevented by a vaccine.

\section{Conclusion}

The overall knowledge of cervical cancer and HPV in this high-risk population was very poor. There is a need to improve the knowledge of this disease among this population. Cervical cancer screening should be made available at little or no cost to them.

\section{Acknowledgements}

This article has been published as part of Infectious Agents and Cancer Volume 5 Supplement 1, 2010: Proceedings of the $12^{\text {th }}$ International Conference on Malignancies in AIDS and Other Acquired Immunodeficiencies (ICMAOI). The full contents of the supplement are available online at http://www.biomedcentral.com/1750-9378/5?issue=S1.

Published: 11 October 2010

doi:10.1186/1750-9378-5-S1-A46

Cite this article as: Anorlu et al: Knowledge of HPV and cervical cancer among HIV-positive women in Lagos, Nigeria. Infectious Agents and Cancer 2010 5(Suppl 1):A46.

\footnotetext{
*Correspondence: rianorlu2004@gmail.com

Lagos University Teaching Hospital, Lagos, Nigeria
} 